\title{
RENDIMIENTO ACADÉMICO Y EVALUACIÓN DOCENTE
}

\author{
ACADEMIC PERFORMANCE AND TEACHER EVALUATION
}

\author{
Miguel A. Vilches, S., ${ }^{1}$ Miguel A. Bustamante U., ${ }^{\star 2}$ Alejandra J. Álvarez $\mathrm{H}^{2}$ \\ 1 Coordinación Curricular. Universidad Autónoma De Chile, Sede Talca, 5 Poniente 1670, Talca, Chile. Correo Electrónico: Miguel.Vilches@Uautonoma.Cl \\ *2 Facultad de Economía y Negocios, Universidad de Talca, Dos Norte 685, 3465548 Talca, Chile. Correo electrónico: mabu@utalca.cl; ale.alvarez.hor@ \\ gmail.com
}

RESUMEN

ABSTRACT

En el presente trabajo se realiza un estudio cuantitativo transversal de carácter exploratorio de los docentes, rendimiento académico y evaluación docente, en el área de formación general de una institución privada de educación superior. La información utilizada fue la totalidad de estudiantes y docentes relacionados (3572). Se aplicó el método de componentes principales, agrupando en 6 factores válidos, correlacionados $(\geq 0,6)$ y con un comportamiento KMO aceptable $(\geq 0,80)$ y que explican un $77 \%$ de la varianza total, alcanzando un alfa de Cronbach total de 0.801 . Entre los hallazgos se determina que la gestión administrativa y eficiencia docente son dimensiones atingente a la evaluación de directores y alumnos. En tanto que, desde la perspectiva del cuerpo docente, los factores con menos peso resultaron ser autoevaluación, cantidad de docentes, edad y ciudad de procedencia. Sin embargo, se observa la necesidad de mejorar el rendimiento académico, mediante la intervención sistemática de los elementos centrales del modelo educativo que combina la transmisión de contenidos como el desarrollo de competencias.

PALABRAS CLAVE: docentes, rendimiento académico, evaluación docente, progresión académica, formación pregrado, gestión administrativa docente, autoevaluación, características personales docentes.
In the present work, a transversal quantitative study of the teachers' exploratory character, academic performance and teacher evaluation is carried out in the general education area of a private institution of higher education. The information used was the totality of students and related teachers (3572). The principal components method was applied, grouping into 6 valid, correlated factors $(\geq 0.6)$ and with an acceptable KMO behavior $(\geq 0.80)$ and that explain $77 \%$ of the total variance, reaching a total Cronbach's alpha of 0.801 . Among the findings, it is determined that the administrative management and teaching efficiency are dimensions relevant to the evaluation of principals and students. While, from the perspective of the faculty, the factors with less weight turned out to be self-evaluation, number of teachers, age and city of origin. However, the need to improve academic performance is observed, through the systematic intervention of the central elements of the educational model that combines the transmission of content such as the development of competences.

KEYWORDS: teachers, academic performance, teacher evaluation, academic progression, undergraduate training, administrative management teaching, self-evaluation, personal characteristics teachers. 


\section{Introducción}

La educación universitaria se encuentra tensionada entre dos modelos curriculares, por un lado el tradicionalista basado en repetición de contenidos orientados en la consecución de objetivos (Guzman, 2015) y el modelo centrado en el aprendizaje, orientado al alcance de resultados de aprendizaje sobre la base de lo que el estudiante es capaz de aprender y demostrar al final de un programa (Chappell y Craft, 2011)

En este último enfoque pedagógico, la institución universitaria tiene el derecho y la obligación (Nonis y Wright, 2003) de transitar hacia un currículum basado en competencias (Guzmán, 2005) cuyo plan curricular responda a las necesidades de la sociedad actual, gestionando una formación integral y flexible con sus estudiantes quienes son llamados a ser agentes activos del aprendizaje (Hernández, Martínez, Juárez y Monroy, 2009) capaces de movilizar y combinar sus recursos, personales y contextuales, para lograr el ejercicio de una actividad en excelencia (Bustamante, Lapo, Oyarzun y Campos, 2017).

El cambio de paradigma curricular (López, Benedito, y León, 2016) establece que la formación por competencias para la vida, sea el foco de la educación en el siglo XXI (Reimers y Chung, 2016) condición que, en el caso de los estudios de pregrado tienen relación directa con el proceso de aprendizaje, cuya eficiencia académica se debe a distintas causas (Navarro, 2003) o factores reflejados en (Garbanzo, 2007) la progresión, tasa de retención y tasa de titulación oportuna. En este sentido Vázquez (2012) explica cómo distintos factores personales, sociales e institucionales son determinantes del nivel de logro de los resultados de aprendizaje.

Como primer factor destacan las decisiones y estrategias metodológicas que los docentes toman al momento de desarrollar el currículum (Jansen y Bruinsma, 2005). Institucionalmente el factor pedagógico, en perspectiva paidocéntrica, centrado en el aprendizaje, conocimientos previos y prácticos (Biggs y Tang 2007) es una necesidad educativa al momento de ejecutar lineamientos provenientes desde el movimiento de reforma curricular de Bolonia que posteriormente fueron contextualizados por el Consejo de Rectores de la Universidades Chilenas (CRUCH, 2013).

La innovación macro curricular busca hacer coincidir la estimación de tiempo total de carga académica con estrategias didácticas exitosas (Glenn y Galán, 2011) a fin de desarrollar seres autónomos, integrales, preparados para la ciudadanía con propósitos claros y habilidades que cimienten el desarrollo de la creatividad intelectualidad y el pensamiento (Sternberg,
1999). Al respecto, Undurraga (2008) profundiza el análisis, incorporando las expectativas de los docentes sobre los estudiantes las cuales además de su formación y experiencia serían predictoras de la eficiencia de los aprendizajes.

En un sentido similar, Perrenoud (2001), destaca el rol del docente como un profesional experto que motiva y gestiona los recursos pedagógicos a fin de guiar el aprendizaje como mediador y estratega del proceso de enseñanza (López, 2002). Trahtemberg (1996) urge, sin embargo, la necesidad de manejar adecuadamente las habilidades sociales por parte de los docentes para poder mejorar el aprendizaje de sus estudiantes. Garbanzo (2007), en cambio menciona determinantes académicos de percepción de los estudiantes sobre los profesores, métodos, ayudas influyen directamente en el rendimiento. En esta perspectiva, los docentes efectivos según Lynn, (2014) serían capaces de motivar el aprendizaje participativo, generando altas expectativas y oportunidades a sus estudiantes para lograr el desempeño esperado (India, 2016).

El rendimiento académico definido como como un indicador del nivel de aprendizaje (Tonconi, 2010), representa un nivel de eficiencia en la prosecución de los objetivos curriculares. García (2008) sin embargo, delimita el rendimiento y condiciona la sostenibilidad y progresión de los estudiantes incluyendo el proceso de evaluación. Guzmán (2012) destaca por su parte que el rendimiento es lo necesario para que el alumno sea capaz de llevar a la práctica sus conocimientos, en tanto que Aldana (2010) destaca algunos determinantes del rendimiento como son el nivel socio económico-cultural, las expectativas de los padres en relación con el rendimiento académico y nuevamente las expectativas de los profesores.

Casuso (2011), hace una profundización al concepto de rendimiento académico desde una perspectiva psicológica, que se corresponde con un estado de bienestar, vigor, absorción y dedicación al estudio en un proceso continuo de desarrollo de capacidades, habilidades y desempeño. Affun (2014) alude al rendimiento en relación con la motivación, poniendo de manifiesto que mejorar el auto concepto de los estudiantes, mejora su motivación interna (Denhart, 2008) y se contrapone a la desmotivación que, Rayan (2000), define como un comportamiento carente de intencionalidad.

Se establece como predictor del rendimiento por una parte (Whitehead, 2007), la percepción del estudiante sobre la sensibilidad del docente y sus expectativas al momento de formar hábitos. Y, por otra, como propone Brow (1998), del atractivo propio de la disciplina, que estimula al estudiante a alcanzar altos niveles de aprendizaje según sus potencialidades, a cuyo respecto, Tella (2007), afirma que esta motiva- 
ción es más perdurable en estudiantes universitarios, especialmente mujeres.

La OECD (2016), establece por su parte, como predictor del rendimiento la relación entre actitudes positivas, unión del trabajo universitario y el aprendizaje, lo cual motiva el rendimiento (Graver, 2010) y que Dell'Angelo (2010), asocia a la intencionalidad o no de las acciones del docente en la sala de clases, las que pueden impactar en sus pupilos estimulando el rendimiento o bien llevando a la baja la dinámica de trabajo, previamente esperada. En este contexto, las directrices de la CNA dan pie a un proceso iniciado el año 2003 por el CRUCH que pone su foco en el aprendizaje de los estudiantes, en los planes de estudio y en el balance de la carga académica, estableciendo las bases hacia la homologación de los procesos formativos y el reconocimiento de competencias (García, 2011; Puig y García, 2007).

Una tercera dimensión, es la evaluación docente por parte de los estudiantes (Poblete, 2016), a cuyo respecto la Unesco (2005), afirma que ser un buen docente implica atender la perspectiva micro curricular, la política de gestión de la docencia así como la presión social respecto de los resultados académicos, todo lo cual provoca un efecto directo sobre la evaluación docente.

\section{Metodología}

El presente trabajo cuantitativo y transversal, adopta primero un carácter exploratorio para validar los reactivos en estudio (Baño, 2016; Kaplan, 2000), luego de componentes principales para generar una estructura de factores interpretable (Freiberg, et al. 2013).

\subsection{Población y muestra}

Para definir el tamaño de la muestra se analizaron las características de los datos, el número de ítems en análisis y la comu nalidad de los ítems (Freiberg, et al. 2013), determinándose un alcance mínimo de 500 observaciones para dar solidez al estudio. (Lloret et al., 2014)

La muestra, para una $\mathrm{p}$ oblación finita (Freiberg, et al. 2013), se determinó mediante un análisis de poder usando el programa $\mathrm{C}^{*}$ Power 3.1 (Faul et al., 2009). Este análisis arrojó un tamaño mínimo de la muestra de 504 casos considerando un alfa de Cronbach $(\alpha=$ $0,05)$, tamaño del efecto $(\mathrm{TE}=0,20)$, una potencia $(1-\beta=0,95)$.

\subsection{Análisis factorial previo de los constructos}

De acuerdo con la metodología de análisis previo sugerida por Baño (2016) y Kaplan (2000), se aplicó análisis de componentes principales (ACP) considerando la matriz de Pearson (Freiberg, et al. 2013) y se asumió el supuesto de normalidad de los ítems (Lloret et al., 2014).

Así mismo, de acuerdo con la metodología de (Freiberg, et al. 2013), se optó por exigir a los reactivos comunalidades altas $(\geq 0,3)$ para asegurar correlaciones fuertes entre ítems y constructos (Bandalos y Finney, 2010) y se exigieron cargas factoriales superiores $(\geq 0,6)$ a fin de excluir reactivos escasamente correlacionados y asegurar una correcta interpretación de las estimaciones multivariantes (Kaplan, 200o).

Se revisó la unidireccionalidad de las escalas y se verificó el número de ítems mínimo ( $\geq 3$ ) para cada constructo (Freiberg, et al. 2013). Además, se evaluó la medida de adecuación $\mathrm{KMO}(\geq 0,80)$, los coeficientes de significancia de Bartlett y se analizó la varianza explicada para obtener la configuración más simple de los constructos (Lloret et al., 2014).

\section{Resultados}

En los resultados se presenta en primer lugar, un análisis descriptivo de los datos analizados a fin de observar el comportamiento de las variables y a continuación de detalla el análisis inferencial de las variables en estudio.

\subsection{Análisis descriptivo}

La muestra del estudio desarrollado tiene las siguientes características:

El cuerpo docente esta compuesto por 36 académicos, cuya edad promedio es de 43 años, teniendo un rango entre 29 y 72 años. El $41 \%$ esta compuesto por varones y el $59 \%$ por mujeres. Según formación académica el 100\% tiene magister y solo 11 de ellos $(30 \%)$ se ubican en el grado de doctor.

La población de estudiantes son 3522. Según rango etario, la edad promedio es de 22, con un rango entre los 18 y 28 años. Según estado civil el $99 \%$ manifiesta un estado de soltería siendo solo 10 personas que tienen estado conyugal (casado o conviviendo).

Según nacionalidad, el 99\% corresponde a chilenos, y el $1 \%$ restante proviene de países como: Colombia, Ecuador y México. Según la historia académica, el egreso del sistema educativo secundario promedio fue el año 2013, con extremos entre 1998 y 2015. El promedio de calificaciones fue de 5,6.

Por otro lado el rendimiento en la prueba de selección universitaria tuvo una media de 534 puntos, una mediana de 532 con una desviación típica de 63 con un rango de puntajes de 293 y 771 ptos.

Según procedencia geográfica el $38 \%$ viven en la ciudad y el $62 \%$ viaja desde alrededores. Considerando los tipos de establecimiento educativos de procedencia, el 51\% corresponde 
a particular subvencionado, 36\% municipal y solo $13 \%$ a colegios particulares. Según financiamiento el $25 \%$ posee beca CAE y Ministerial.

\subsection{Componentes principales}

Se realizó un análisis inferencial de carácter exploratorio de los datos mediante el método de componentes principales, se aplicó rotación varimax con Kaiser, alcanzando convergencia en 10 iteraciones de las 25 previstas.
Se determinó que los registros son pertinentes para el análisis factorial, de acuerdo con el índice KMO que alcanzó un resultado de o,715 considerado adecuado (Tabla 1 ).

La Tabla 1, muestra los factores y sus respectivos componentes, los que como se observa, presentan altas cargas factoriales. Se determinaron 6 factores que agrupan un total de 17 variables observables y que explican un $77,63 \%$ de la varianza con un alfa de Cronbach de 0,807 , considerado bueno.

Tabla 1: Factores determinados

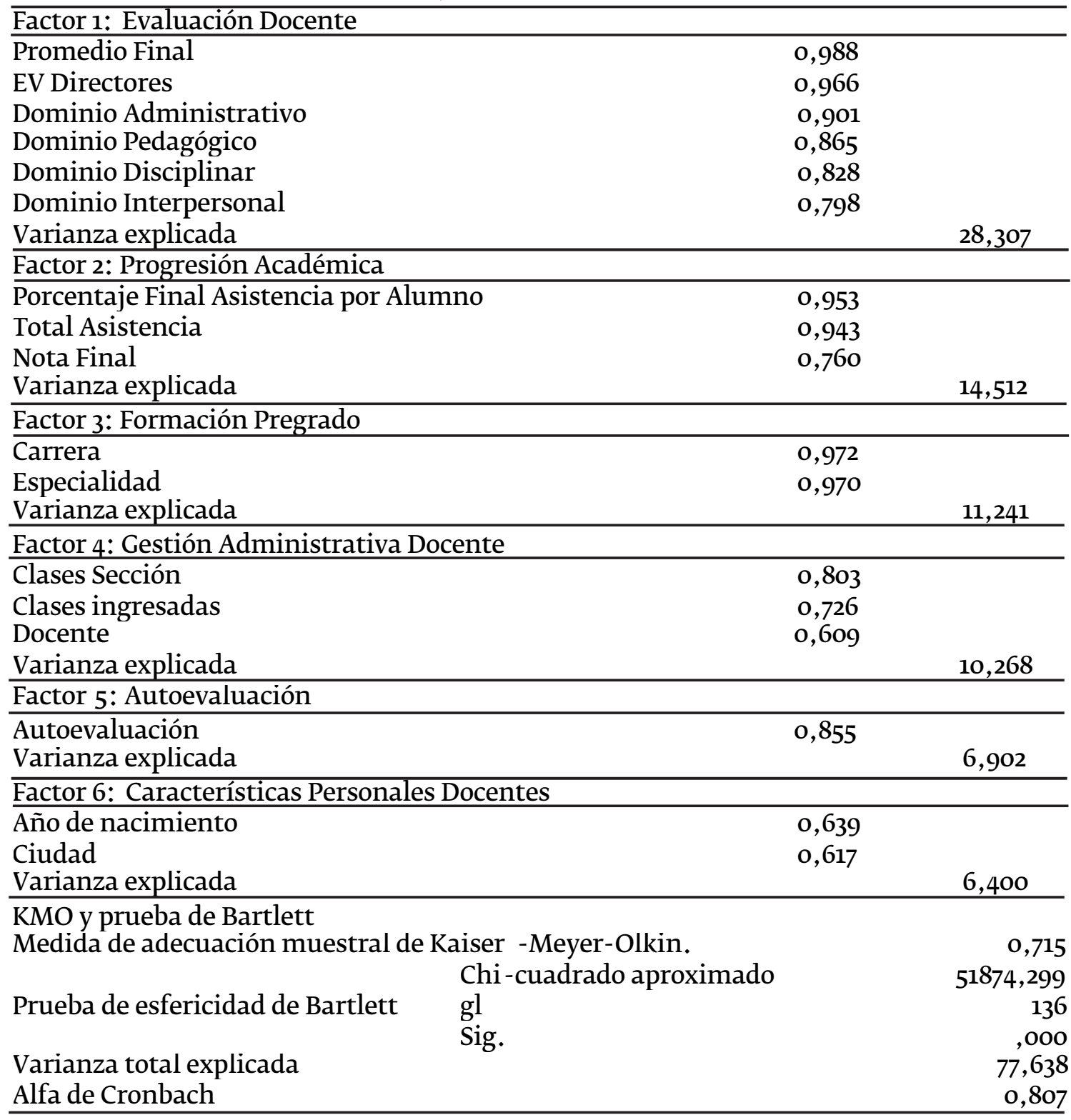

El primer factor, Evaluación docente, se corresponde con un procedimiento regular y sistemático desarrollado semestralmente y que explica la varianza en un $28,3 \%$. Ha sido dividido en cuatro niveles de desarrollo de las competencias docentes, siendo los académicos pertenecientes a la muestra clasificados como
Destacados, quienes obtienen un porcentaje de logro promedio superior a un $89 \%$. Los indicadores relacionados a este factor aluden a la percepción de la calidad que tienen los directores de carrera sobre el compromiso con la docencia y el cumplimiento de las obligaciones académicas. El componente de la evaluación 
directiva, al interior del sistema de evaluación docente, pondera un $40 \%$ del total.

Los dominios definidos como administrativo, pedagógico, disciplinar e interpersonal pertenecen a la evaluación docente realizada por estudiantes que pondera un $40 \%$ al interior del sistema de evaluación docente. En particular, destaca la dimensión administrativa relacionada con la planificación, evaluación, compromiso, resultados, cumplimiento de horarios y uso de herramientas pedagógicas. En segundo lugar, el se encuentra la dimensión pedagógica, que integra la forma de hacer clases, evaluar y retroalimentar el desempeño. El dimensión disciplinar, en tercer lugar, se refiere al trabajo de clase, la vinculación con el perfil de egreso, actualización teórica y relación con el mundo laboral. En cuarto lugar, queda el dominio interpersonal, que incluye las normas, climas de aula, atención a la diversidad, acogida positiva y trabajo colaborativo.

El segundo factor, Progresión Académica, se define en la institución como el avance de los estudiantes en el plan de estudios y que explica un $14,5 \%$ de la varianza. El área de Formación General (FG), en paralelo se integra a la formación profesional y disciplinar. Ésta área, se compone de 10 electivos que a su vez tributan a las competencias genéricas de la institución. Cada una de las asignaturas de FC dispuestas en el plan de estudio, poseen 3 créditos y se replican en cada semestre de los ciclos formativos que atraviesan todos los estudiantes. Las asignaturas no tienen prerrequisito.

Por otra parte, los parámetros metodológicos y evaluativos están delimitados por diferentes resoluciones y reglamentos de evaluación que para el caso de la asistencia, estipula una exigencia diferenciada desde un $70 \%$ en el ciclo inicial a un $50 \%$ ciclo avanzado y final.

Por su parte, la calificación de aprobación para todas las asignaturas en las distintas áreas formativas está fijada en el estándar 4,0 dando la opción de quienes tengan menos de un 3.94 a una evaluación recuperativa. El promedio lineal de los estudiantes fue, a la fecha del presente trabajo, de un 5.4 con una asistencia de $79 \%$ y aprobación de un $94 \%$. Los trabajos son evaluados en el proceso mismo y se observa que los estudiantes se muestran motivados por asistir y dar cuenta del nivel de logro de cada uno de los productos curriculares comprometidos.

El tercer factor, Formación Pregrado, explica el $11.2 \%$ de la varianza. En general se observa que la institución no hace distinciones metodológicas ni evaluativa para las 23 carreras el área de formación general, ya que el área se ejecuta en paralelo a las asignaturas disciplinares y profesionales, dotando al estudiante de las competencias distintivas y estructurales previstas en el perfil de egreso. En consecuen- cia, en los distintos itinerarios formativos, las asignaturas se comprometen a desarrollar competencias genéricas transversales (Habilidades de la Comunicación, Compromiso ético, trabajo en Equipo, pensamiento crítico y responsabilidad social empresarial) incluidas en cada uno de los perfiles de egreso, sin embargo, cada curso está centrado en un resultado de aprendizaje, que consecutivamente se va desarrollando mediante la evaluación y uso de instrumentos criteriales.

El cuarto factor, Gestión Administrativa Docente, consiste en indicadores propios de la gestión de procesos docentes y explica en un $6,9 \%$ la varianza total. En la sede objeto de este estudio, es tutelada por la unidad respectiva, que en perspectiva del del PDE tiene indicadores específicos a los cuales debe responder. En este contexto, el área tiene sobre un $97 \%$ de clases planificadas y desarrolladas para un universo de 37 docentes. La información destaca por ser un elemento evidente pero a la vez indispensable de los demás indicadores, y se constituye en la base de la gestión que tributa al funcionamiento habitual de la universidad como un todo e incidiendo, en conjunto, en un 10\% del sistema de gestión global.

El quinto factor, Autoevaluación, explica un 6,9 la varianza y se lleva a efecto al interior del sistema de evaluación docente, ponderando el $20 \%$ del sistema general de gestión. Cada uno de los académicos, habiendo desarrollado dos tercios de su planificación, realizan un proceso de reflexión sobre distintas dimensiones del programa de enseñanza - aprendizaje. Los ámbitos por evaluar son: pedagógica, interpersonal, administrativa, conocimiento disciplinar y gestión del trabajo autónomo para así, ponderar un solo nivel de logro. De la totalidad de los docentes, la cobertura fue de un $100 \%$ y el nivel de logro rue de un $96 \%$, catalogándose los docentes como destacados.

El sexto factor, Características personales docentes, consiste en la edad y las ciudades de procedencia que explican un 6,4 de la varianza total. El cuerpo académico esta constituido por 37 docentes, que en promedio tienen 43 años, un $90 \%$ son magister y el $10 \%$ restante tiene doctorado. Las ciudades de procedencia son: Talca, Santiago y Concepción.

\section{Discusión}

La metodología empleada, análisis de componentes principales, agrupa 6 factores que explican un $77,63 \%$ de la varianza de la muestra aludida (3572 sujetos). La descripción de estos factores, sus características, en relación con la teoría demuestran distintas perspectivas sobre la temática; Docentes, Rendimiento académico y Evaluación Docente. Estudios similares se han realizado, identificando también factores pero avanzando hacia el análisis confirmatorio 
de los mismos (Montenegro y González, 2013). Una primera arista se encuentra en la incidencia de la evaluación de los directivos sobre los académicos. Este ejercicio heteroevaluativo, se orienta a evidenciar el compromiso con la docencia y cumplimiento de las obligaciones académicas y se expresa en distintos niveles de logro (Destacado, Competente, Básico e insatisfactorio). La evaluación de dirección de carrera, en teoría pondera un $40 \%$, pero para efectos prácticos tiene una incidencia superior a la evaluación de los estudiantes o la misma autoevaluación docente. Esta condición se difiere de Lynn (2014) y Whitehead (2007) quienes respectivamente apelan a la sensibilidad, expectativas y formación de hábitos para así motivar, generando expectativas y oportunidades a los estudiantes. La aprobación laboral por parte de la dirección, incide a un nivel significativo alto en los mismos docentes, su eficiencia académica y por ende, en sus resultados del proceso. En este contexto, la educación puede estar en cualquier extremo curricular, o en tránsito, pero seguirá siendo fundamental como evalúan el desempeño docente por sobre la propuesta de Perrenoud (2001) sobre el experto que motiva y gestiona recursos pedagógicos.

Un segunda arista se encuentra en la asistencia y rendimiento académico de los estudiantes, lo que se compara con estudios de la eficacia de la formación en habilidades cooperativa de estudiantes universitarios (Lidón, et al., 2017).

La teoría indica que asistir a clases que no es condicionante del rendimiento, pero sí es un apoyo para el desarrollo de los productos educativos que se desprenden desde los resultados de aprendizaje (OECD 2016) pudiendo unir con esto el saber académico con elementos prácticos del mundo laboral. En este punto concordando con Guzmán (2012) referente al rendimiento, es necesario que el alumno sea capaz de llevar a la práctica sus conocimientos, por lo mismo, la asistencia es un referencial en un contexto de desarrollo de capacidades, habilidades y desempeño (Casuso, 2011) las cuales mediante demostración y constante feed back los estudiantes pueden mejorar incluso su motivación (Denhart, 2008) siendo más perdurable en estudiantes que un mero impulso extrínseco ( $79 \%$ asistencia final y 94\% aprobación).

En esta misma línea de trabajo, se ha analizado la calidad de la tutoría académica universitaria mediante análisis factorial (Quispe, et al., 2017), determinándose que la tutoría por parte del estudiante depende del tipo de tutoría que recibe por parte del docente y que los estudiantes tienen mayor preferencia por la tutoría individualizada que la grupal y virtual. Como es el caso del presente estudio dónde la formación general, es un área de formación transversal, relevante para definir la pertinencia de un programa, como ya ha sido analizado en otros estudios similares (Almonacid, et al., 2009), en los cuales se ha concluido la pertinencia, mediante análisis factorial, de dicha pertinencia, en la cual importa más el desempeño, individual, que la disciplina y que, por lo mismo, se despliega un sistema de monitoreo en todas las mallas curriculares con una carga de créditos constante.

Una tercera arista, relacionado con los docentes, su reflexión de desempeño (autoevaluación) tienen una incidencia significativa, es decir, pero comparada con las demás solo explica la varianza en un $6 \%$. Tradicionalmente un académico debe ser alguien capaz mediante la reflexión de lograr las adecuaciones curriculares necesarias para conseguir las competencias comprometidas en las asignaturas que desarrolla. Desde esta misma perspectiva, Fernández et al., 2015, en su estudio evidenció el predominio del enfoque centrado en el cambio conceptual, donde los docentes con menos años de experiencia mostraron una tendencia a la utilización de un enfoque centrado en la transmisión de conocimientos, precisamente opuesto al enfoque de enseñanza centrada en competencias, que se relaciona con esta dimensión más bien supeditada a un contexto micro curricular estructurado (diseño instruccional) que en concordancia con la Unesco (2005), afirma que ser un buen docente implica atender la perspectiva micro curricular puesto que la enseñanza va más allá de la transmisión de contenidos propiciándose cada vez más el desarrollo de competencias (Hamodi, et al., 2018), más allá de tan sólo atender la presión social respecto de los resultados académicos.

A modo de reflexión se observa que asistencia y rendimiento permiten comprobar que también, otras áreas debieran ser sometidas a un modelo de verificación similar, como puede ser el desarrollo de habilidades de emprendimiento (López, et al., 2017), compatible con el modelo disciplinar - profesional de desarrollo de habilidades, generando nuevos lineamientos de investigación en otras dimensiones de formación a nivel universitario.

\section{Conclusiones}

Del análisis se aprecia que los factores reiteran una situación académica tradicional, es decir, gestión administrativa y eficiencia docente son dimensiones atingente a la evaluación de directores y alumnos.

Se aprecia así mismo, que la asistencia, carrera y clases planificadas tienen mayor incidencia que la habilidad interpersonal, autoevaluación y características de los docentes. Desde la perspectiva del cuerpo docente, los factores con menos peso resultaron ser autoevaluación, cantidad de docentes, edad y ciudad de procedencia. 
Se observa además el tránsito desde un modelo educativo tradicional que avanza incorporando en su estructura nuevos elementos curriculares y administrativos que sirvan para mejorar el rendimiento académico, mediante la intervención en los elementos centrales del modelo educativo que combina la transmisión de contenidos como el desarrollo de competencias.

\section{Referencias}

Affum-Osei E (2014) Achievement motivation, academic self-concept and academic, achievement among high school students. European Journal of Research and Reflection in Educational Sciences Vol. 2 No. 2, 2014.

Aldana, K., Reyna, R y Ayolaida, M (2010) Visión del desempeño académico estudiantil en la Universidad Contraccidental Lisandro Alvarado, en revista compendium vol 13 , nun 24 . Universidad Centroccidental Lisando Alvarado, Venesuela 2010. Pp 5-21

Almonacid, Paula; Montes, Isabel Cristina; Vásquez, Juan José Un análisis factorial para evaluar la pertinencia de un programa académico desde la perspectiva de los graduados: un estudio de caso Ecos de Economía, vol. 13, núm. 29, octubre, , pp. 97-126 (2009).

Artunduaga, $M$ Variables que influyen en el rendimiento académico en la Universidad. [Internet]. Madrid: Departamento: Métodos de Investigación y Diagnóstico en Educación (2008).

Bandalos, D. L. y S. J. Finney, Factor Analysis: Exploratory and Confirmatory. En G. R. Hancock y R. O. Mueller (Eds.), Reviewer's guide to quantitative methods. Routledge: New York. (2010).

Baño D. J., E. P. Villacrés, L. F. Arboleda, T. García. Evaluación del clima organizacional del personal docente de las Instituciones de Educación Superior (IES) de la provincia de Chimborazo - Ecuador. doi.org/10.15381/idata.v19i2.12816. Revista Industrial data 19(2):59-68 (2016).

Biggs, J., y Tang, C. Teaching for quality learning at university: what the student does (3nd ed.). Phildelphia: Society for Research into Higher. Education. Open University Press. (2007).

Brown, S., Armstrong., S., y Thompson, I Motivating Students. London: Kogan Page Limited. (1998).

Bustamante, M, . Lapo, M, Oyarzún, C., y Campos, M. Análisis de la Percepción del Docente en Tres Universidades Chilenas tras la Implementación del Currículum Basado en Competencias. Formación universitaria, 10(4), 97-110 (2017).

Casuso, M. Estudio del estrés, engagement y rendimiento académico en estudiantes universitarios de Ciencias de la Salud. Tesis doctoral, Universidad de Málaga. (2011).

Chappell, K., y Craft, A Creative learning conversations: producing living dialogic spaces. Educational Research 56. (2011).

Consejo de Rectores de las Universidades Chilenas (CRUCH 2013) Manual para la implementación del Sistema de Créditos Académicos TransferiblesSCT - Chile. Santiago de Chile.

Dell'Angelo, T. Teacher trust and perceived obstacles as mediators of student achievement in Philadelphia high schools. ProQuest Information \& Learning, p. 7o. (2010).
Denhardt, B., Denhardt, V., Y Aristigueta, P. Managing, Human Behavior in Public and Nonprofit Organizations. Sage. Publications, Inc. Journal of Personality and Social Psychology, 47, 944-952. (2008).

Edel, R El Rendimiento Académico: Concepto, Invesıtigación y Desarrollo. Revista Electrónica Iberoamericana sobre Calidad, Eficacia y Cambio en Educación, 1(2). (2003).

Faul F., E. Erdfelder, A. Buchner y A. G. Lang, Statistical Power Analyses using $G^{*}$ power 3.1: Tests for Correlation and Regression Analyses, Behav Res Methods, 41(4) 1149-1160 (2009).

Fernández Castillo, Evelyn, García Ortiz, Yaritza y Torres López, Raciel. Análisis factorial y confiabilidad del Cuestionario de Enfoques de Enseñanza. EDUMECENTRO, 7(4), 146-161 (2015) .

Fernando, R., y Connie, C (editores), México, Fondo de Cultura Económica, (2016).

Freiberg H. A., J. B. Stover, G. de la Iglesia, M. Fernández, Polychoric And Tetrachoric Correlations In Exploratory And Confirmatory Factorial Studies. Prensa Médica Latinoamericana - ISSN 1688-4094 Ciencias Psicológicas, VII (2): 151 - 164 (2013).

Garbanzo, G. Factores asociados al rendimiento académico en estudiantes universitarios, una reflexión desde la calidad de la educación superior pública. Revista Educación 31, 43-63. (2007).

Garbanzo, M. Factores asociados al rendimiento académico en estudiantes universitarios, una reflexión desde la calidad de la educación superior pública. Revista Educación, 31(1), 43-63. (2007).

García, B. Las dimensiones afectivas de la docencia. Universidad autónoma de México: Revista Digital Universitaria. 10, 1-14. (2009).

García-Cabrero, B., Loredo, G. Análisis de la práctica educativa de los docentes: pensamiento interacción y reflexión, Revista Electrónica de Investigación Educativa, Especial, Vol. 10 (2008).

Glenn, C., y Galan, A. Educational Freedom and Accountability for School Quality in Spain. International Journal for Education Law and Policy (IJELP), 7, 1-2. (2011).

Graber, R. Factors that are predictive of Student achievement outcomes and an analysis of these factors in high-poverty schools versus low-poverty schools. ProQuest Information \& Learning, p. 71. (2010).

Guzmán, M. Desarrollo de competencias en la formación inicial de profesores. Riesgos y desafíos. Revista Pensamiento: La problemática de las competencias en la perspectiva de la calidad de la educación, 36, pp.192-203. (2005).

Guzmán, M., Maureira, O., Guzmán, A y Vergara, A Innovación curricular en la educación superior. ¿Cómo se gestionan las políticas de innovación en los (re)diseños de las carreras de pregrado en Chile? En Perfiles educativos vol.37 no 149 (2015).

Guzmán, M.. Modelos predictivos y explicativos del rendimiento académico universitario: caso de una institución privada en México. Madrid: Universidad Complutense de Madrid. (2012)

Hamodi Galán Carolina, Juan Antonio Moreno-Murcia, Raúl Barba Martín. Medios de Evaluación y Desarrollo de Competencias en Educación Superior en Estudiantes de Educación Física. Estudios Pedagógicos XLIV, N² 2: 241-257, (2018).

Hernández, F., Martínez, P., Martínez, M., y Monroy Hernández, F.. Aprendizaje y Competencias. Una nueva mirada. Revista Española de Orienta- 
ción y Psicopedagogía, 20 (3), 312-319. (2009).

India, M Factors Affecting the Academic Achievement: A Study of Elementary School Students of NCR Delhi, , Ph.D Assistant Professor, College of Teacher Education (MANNU), NUH Mewat, Haryana. Vol.7, No.4, (2016).

Jansen, P., y Bruinsma, M. Explaining achievement in higher education. Educational Research and Evaluation. 11, 235-252. (2005).

Kaplan, D., Structural Equation Modeling. Foundations and Extensions. Thou-sand Oaks, CA: SACE (2000).

Lidón Moliner Miravet, Arecia Aguirre García-Carpintero, Ana Domenech Vidal, Teresa Vallet Bellmunt, Ilu Vallet Bellmunt, Francisco Alegre Ansuategui. Diseño, validación y análisis factorial exploratorio y confirmatorio de la escala de actitud Cohesiona para la evaluación de la eficacia de los talleres de habilidades cooperativas. Estudios Pedagógicos XLIII, $\mathrm{N}^{\circ}$ 1: 213-234, (2017).

Lloret-Segura, S., A. Ferreres-Traver, A. Hernández-Baeza e I. Tomás-Marco, El análisis factorial exploratorio de los ítems: una guía práctica, revisada y actualizada. Anales de psicología, 30(3), 1151-1169 (2014).

López Torres Virginia Guadalupe, Luis Ramón Moreno Moreno, Sósima Carrillo. Enseñanza del emprendimiento en la educación superior (diseño de una escala, análisis factorial y confiabilidad). Revista Universitaria de Administración. Vol. 8, Núm. 14 (2017).

López, C., Benedito, V., y León, J. El Enfoque de Competencias en la Formación Universitaria y su Impacto en la Evaluación: La Perspectiva de un Grupo de Profesionales Expertos en Pedagogía. Formación universitaria,9(4), 11-22 (2016).

López, F. (202) El análisis de contenido como método de investigación, Revista de Educación, Universidad de Huelva, 167-179 (2002).

Lynn, M Risk Factors for Adolescent, Academic Achievement. Wisconsin Family Impact Seminars. (2010).

Montenegro Maggio Helena, Carlos González Ugalde. Análisis factorial confirmatorio del cuestionario, Enfoques de Docencia Universitaria. Estudios Pedagógicos XXXIX, № 2: 213-230, (2013).

Nonis, P., y Wright, D Moderating effect of achievemente strinving an situational optimis on the relationships betwwen ability and performance outcomes os college students. Rsearch in Higher Education, $44327-346$ (2003).

OECD Low performing students: Why they fall behind, and how to help them succeed. PISA. Paris: Author. (2016).

Perrenoud, P La Formación de los Docentes en el Siglo XXI, Revista de Tecnología Educativa, Santiago de Chile, XIV (3) 503-523. (2001).

Poblete, F Evaluación docente: la importancia de la percepción de los estudiantes. (2017).

Quispe Fernández Gabith Miriam, Dante Ayaviri Nina, Otto Eulogio Arellano Cepeda. Aplicación del Análisis Factorial en la medición de la calidad de la tutoría académica universitaria. Revista Espacios. Vol. 38 (№ 50). Pág. 16. (2017).

Ryan, M., Deci, L Self-determination theory and the facilitation of intrinsic motivation, social development, and well-being. American Psychologist, 55, 68-78 (2000).

Sternberg, R Estilos de pensamiento. Claves para identificar nuestro modo de pensar y enriquecer nuestra capacidad de reflexión. Barcelona: Paidós. (O esto: Almansa Martínez, Pilar. (2012). Qué es el pensamiento creativo.Index de Enfermería, 21(3), 165-168. (1999).

Tella A. The impact of motivation on students' academic achievement; and learning outcomes in mathematics among secondary school students in Nigeria. Eurasia Journal of Mathematics, Science and Technology Education 3(2), pp 149-55 (2007).

Tonconi, J. Factores que influyen en el rendimiento académico y la deserción de los estudiantes de la facultad de ingeniería económica de la una-puno, periodo 2009. Cuadernos de Educación y Desarrollo - EUMED, 23. (2010).

Trahtemberg, L. Educación para el tercer milenio. Lima: Editorial Bruño. (1996).

Unesco. Protagonismo docente en el cambio educativo. Oficina regional de educación para América Latina y el Caribe. (2005).

Vázquez, C. Decimoséptimas Jornadas "Investigaciones en la Facultad" de Ciencias Económicas y Estadística. Noviembre de 2012. Instituto de Investigaciones Teóricas y Aplicadas. Escuela de Contabilidad. (2012).

Whitehead, O. African-American students'perceptions of teacher attitudes on academic achievement and discipline sanctions. ProQuest Infor- 